\title{
14. The Condition of Appearance of the Regular Slow Wave Sequence in the Hippocampus of the Dog.
}

\author{
Y. Shimazono \\ Dept. of Neuropsych., Kanazawa Univ. School of Med.
}

1) Two types of regular slow waves are demonstrated in the dog's hippocampus in relation to the animal's behaviors. One of them, the hippocampal rhythmic wave-S, is produced only after the sleep pattern, sometimes spontaneously and sometimes by optimal stimuli. While the wave is maintained, the animal remains in the sleeping posture, but shows occasional localized twitches of eyelids, conchae, vebrissae, some muscles etc. and frequently rapid movement potentials of eyeballs. The other, the hippocampal rhythmic wave-A, is seen as a short train during wakefulness and is accompanied by some bodily action of the dog, e.g. head-turning, sniffing and exploring the floor.

2) We did not succeed in inducing the long-lasting slow wave sequence in the hippocampus or other subcortical regions by electrical stimulation of any subcortical structure in chronic dogs in the fully awakened state, although we could produce short trains of the waves by direct electrical stimulation of some muscles of the extremities.

3) When a dog having chronically implanted electrodes is fixed to the table under ether anesthesia and put under curarization, long series of very regular, slow waves quite similar to the rhythmic wave-S of the experiment with the chronic dog without anesthesia are observed in the hippocampus and some other subcortical regions.

At first, this phenomenon was supposed to be due to curarization, because in earlier experiments this regular pattern was found to disappear when the immobilizing effect of curare grew weaker and to reappear after re-administration of natural or synthetic curares. But in later experiments this action of curare could not always be confirmed. The effect of ether is quite noticeable also especially when the anesthesia has weakened.

4) In the acute experiments, somewhat irregular slow waves can often be seen in the hippocampal region, and the continuous series of regular slow waves are produced by electrical stimulation of subcortical structures. This regular pattern is also produced by low-frequency or weak high-frequency stimulation of the sciatic nerve but is desynchronized when the stimulation is too strong. In the later stage of the acute experiment, the regular slow waves appear only during stimulation and the pattern is not desynchronized by increasing the strength of the stimulus.

5) From these findings, we conclude that the long-lasting hippocampal slow waves appear more readily when the hippocampus is moderately activated while in the altered state, perhaps that of lowered activity due to whatever cause.

(This study is performed with Drs. T. Horie, N. Hori, S. Chikazawa, M. 
Okabe and Y. Yanagisawa.)

\title{
15. From the Standpoint of Psychiatry.
}

-Particularly on the Working Mechanism of Psychosurgery-

\author{
S. HIROSE
}

Dept. of Psy. Nippon Med. School

Recently, on the behavior as brain function, the interrelation between emotional drives and the limbic systems is discussed. But there is self-consciousness in human beings. I think it may be that the frontal lobes are the seat of self-consciousness which is active in shaping an upper sphere of personality that gives structure and direction to emotional drives.

Previously, as to lobotomized personality traits I have analized and synthesized the following. (1) Reduction of drive and emotion. (2) Simplicity and shallowness of feeling. (3) Blunting of sensitivity. (4) Reduction of interest to self-consciousness. (5) Lack of restraint. (6) Lack of spontaneity and initiative. (7) No influence from pre-operative memory, intelligence and habitual behavior.

FULTON and others speculate on the working mechanism of psychosurgery in the anatomical and physiological knowledge, but $I$ think that the actual results on human patients are much more complicated.

Since 1957, I devised a new method of orbito-ventromedial undercutting for the purpose of bringing about maximum therapeutic effects while minimizing postoperative complications and unfavorable personality changes. The working mechanism of the new method is very complicated, but $I$ think that the quantitative factors of operation, such as the amount of cortex to be separated, and the qualitative factors, such as the site of operation, always accompany each other in different degrees and different combinations.

\section{Effects of Hippocampal Ablation on Behavior in the Rat.}

\section{H. NIKI}

Inst. of Brain Resserch, Dept. of Psychol. Univ. of Tokyo

A series of experiments was conducted with white rats to investigate the effects of hippocampal ablation upon avoidance response, maze performance, brightness discrimination, stripe discrimination, and general activity.

The hippocampus was ablated bilaterally through neocortex by suction. Histological study of the brains showed that a part of the hippocampal tissue 